\title{
Determination of isoprostane 8-iso-PGF2 $a$ and prostaglandin GF2 $a$ in plasma and intestine of specific-pathogen-free chickens challenged with Eimeria maxima
}

\section{Victor M. Petrone-Garcia}

Programa de Doctorado en Ciencias de la Salud y Producción Animal, Facultad de Estudios Superiores Cuautitlan, Universidad Nacional Autonoma de Mexico

Raquel Lopez-Arellano

Laboratorio No 5: LEDEFAR, Unidad de Investigacion Multidisciplinaria, Facultad de Estudios Superiores Cuautitlan, Universidad Nacional Autonoma de Mexico

\section{Gabriela Rodríguez Patiño}

Laboratorio No 5: LEDEFAR, Unidad de Investigacion Multidisciplinaria, Facultad de Estudios Superiores Cuautitlan, Universidad Nacional Autonoma de Mexico

\section{Miriam A. Castillo Rodríguez}

Laboratorio No 5: LEDEFAR, Unidad de Investigacion Multidisciplinaria, Facultad de Estudios Superiores Cuautitlan, Universidad Nacional Autonoma de Mexico

\section{Daniel Hernandez-Patlan}

Division de Ingenieria en Nanotecnologia, Universidad Politecnica del Valle de Mexico

\section{Bruno Solis-Cruz}

Division de Ingenieria en Nanotecnologia, Universidad Politecnica del Valle de Mexico

\section{Xochitl Hernandez-Velasco}

Departamento de Medicina y Zootecnia de Aves, Facultad de Medicina Veterinaria y Zootecnia, UNAM

\section{Fernando Alba-Hurtado}

Departamento de Ciencias Biologicas, Facultad de Estudios Superiores Cuautitlan, Universidad Nacional Autonoma de Mexico

\section{Christine N. Vuong}

Department of Poultry Science, University of Arkansas

\section{Inkar Castellanos-Huerta}

Programa de Doctorado en Ciencias de la Salud y Producción Animal, Facultad de Medicina Veterinaria y Zootecnia, UNAM

\section{Guillermo Tellez-Isaias ( $\square$ gtellez@uark.edu )}

Department of Poultry Science, University of Arkansas 


\section{Research Article}

Keywords: chickens, Eimeria maxima, isoprostane 8-iso-PGF2a, PGF2a, intestine, prostaglandin Posted Date: March 15th, 2021

DOI: https://doi.org/10.21203/rs.3.rs-289590/v1

License: (c) (i) This work is licensed under a Creative Commons Attribution 4.0 International License. Read Full License 


\section{Abstract}

The purpose of this study was to evaluate and determine the concentration of isoprostane 8-iso-PGF2a and prostaglandin GF2a (PGF2a) from plasma and intestine in specific pathogen-free (SPF) chickens challenged with Eimeria maxima (EM) using solid-phase microextraction and ultra-performance liquid chromatography/tandem mass spectrometry. Forty one-day-old male SPF chickens were randomly allocated to one of two groups with four replicates ( $n=5$ chickens/replicate). Groups consisted of Control (no challenge) or the Challenge group EM (40,000 sporulated oocysts/bird). At day 7 and 9 postchallenge, half of the chickens were euthanized in both groups to determine plasmatic and enteric concentrations of isoprostane 8-iso-PGF2 $a$ and PGF2a. Enteric levels of both 8-iso-PGF2 $a$ and PGF2 $a$ were significantly increased at 7 (8-iso-PGF2a $P=0.0000252$; PGF2 $a \mathrm{P}=0.00000268$ ) and 9 days (8-iso$P G F 2 a P=0.000000717$; PGF2 $a P=0.00000222)$ post-challenge compared to non-challenge control chickens. However, plasma levels of isoprostane 8-iso-PGF2 $a$ and PGF2a were similar in both groups. A significant reduction $(P=0.0000095)$ in oocyst excretion was observed in chickens at 9 days postchallenge compared to 7 days. Chickens challenged with EM showed an inflammatory response associated with significant increases in enteric PGF2 $a$ and 8-Iso-PGF2a, suggesting that the active disease phase was accompanied by inflammation and oxidative stress within the intestinal layer.

\section{Introduction}

Coccidiosis is a parasitic enteric disease of animals caused by coccidian protozoa from the Apicomplexa phylum. In a recent study, the global cost of coccidiosis in broiler chickens was estimated at $\sim £ 10.36$ billion ${ }^{1}$. Eimeria spp. have a remarkable and complex life cycle, including sexual and asexual reproduction with intracellular and extracellular phases $2,3,4$. Hence, during the disease, the gut-associated lymphoid tissues respond with a series of innate and acquired immune reactions against the parasite ${ }^{5,6}$. Several investigators have extensively studied and documented the immunopathology of cellular responses involving the secretion of pro-inflammatory cytokines to Eimeria infections in chickens ${ }^{7,8,9,10,11,12}$. However, little is known about the role of prostaglandins $(P G)$ and isoprostanes $\left(F_{2^{-}}\right.$ Ips) as part of the innate response during clinical coccidiosis. Prostaglandins are a group of lipid compounds from the eicosanoid family implicated in inflammation, allergy, fever, and other immune responses that are generated from arachidonic acid by the action of cyclooxygenases (COXs) isoenzymes. On the other hand, $F_{2}$-Ips are PG-like complexes formed from free radical catalyzed oxidation of arachidonic acid, without the action of COXs. The measurement of $F_{2}-I p s$, especially 8-epi$\mathrm{PGF}_{2 a}$, is recognized as a consistent biomarker of lipid peroxidation and is currently used as a sensitive index of oxidative stress in vivo.

The purpose of this study was to evaluate and determine the concentration of prostaglandin GF2a (PGF2a) and isoprostane 8-iso-PGF2 $a$ in plasma and intestine of specific pathogen-free (SPF) chickens challenged with Eimeria maxima using solid-phase microextraction and ultra-performance liquid chromatography/tandem mass spectrometry. 


\section{Results}

The evaluation of isoprostane 8-iso-PGF2 $a$ and PGF2 $a$ from jejunum and plasma in SPF chickens challenged with Eimeria maxima at 7- and 9-days post-challenge are summarized in Table 1. Enteric levels of both isoprostane 8-iso-PGF2a (7 days: $P=0.0000252 ; 9$ days: $P=0.000000717)$ and PGF2a (7 days: $P=0.00000268$; 9 days: $P=0.0 .00000222)$ were significantly increased $(P<0.01)$ at both time points compared to the non-challenge control chickens (Table 1; Figure 2). However, plasma levels of isoprostane 8-iso-PGF2a (7 days: $P=0.29812 ; 9$ days: $P=0.2923$ ) and PGF2a (7 days: $P=0.021489$; 9 days: $P=0.0403$ ) were similar ( $P>0.01$ ) in both groups (Table 1; Figure 3 ). Table 2 shows the results of $E$. maxima OPG of feces from the SPF chickens at 7- and 9-days post-challenge. As expected, challenged chickens showed a significant increase ( 7 days: $P=0.00000095$; 9 days: $P=0.00000095$ ) in oocyst excretion when compared with non-challenge control chickens. Moreover, a significant reduction $(P<0.05)$ in oocyst excretion was observed in chickens at 9 days post-challenge compared with day $7(P=0.00002)$ (Table 2).

\section{Discussion}

In addition to the critical job of absorbing water and nutrients, enterocytes also play an essential role in the mucosal immune response, maintaining tolerance to beneficial microbiota, and identifying luminal pathogens. The invasion of Eimeria spp in intestinal epithelial cells is a complex process that includes several events beginning with the excystation of sporozoites after oral ingestion of the oocysts ${ }^{13,14}$. As intracellular parasites, attachment and invasion of the sporozoites to the host cell is recognized by Tolllike receptors 4 and 15, involved in pathogen recognition and activation of the mucosal inflammasome IL-1/IL-18 axis, which is responsible for recruiting and activating heterophils, natural killer cells, mast cells, macrophages, and the transcription factor NF-KB ${ }^{15,16,17,18}$. Nevertheless, sporozoites have evolved a unique molecular system fueling motility and invasion of epithelial cells through gliding motility, allowing them to rapidly invade host cells and form an intracellular parasitophorous vacuole that protects them from the intracellular hostile environment ${ }^{19,20,21,22}$. Within this vacuole, these Apicomplexa parasites gain precious time to continue with their multifaceted life cycle. Each phase of the sexual, asexual, intracellular, or extracellular stages of this prehistoric and remarkable parasite are associated with severe local inflammation, autophagy, apoptosis, cellular death, hemorrhages, and necrosis in the intestinal mucosa $20,21,23,24,25$. Hence, coccidia infections are characteristic by inducing chronic inflammation and excessive tissue damage because of the parasite infection and the host immune response elicited against the invaders. In chickens, macrophages are the primary sources of nitric oxide, superoxide, and hydrogen peroxide as essential mediators of both innate and acquired immunity, thus increasing during coccidia infections $26,27,28,29,30$. In the present study, chickens challenged with $E$. maxima showed a significant increase $(P<0.01)$ in enteric PGF2a at 7- $(P=0.00000268)$ and 9 -days $(P=0.00000222)$ postchallenge when compared with non-challenge chickens. However, the serum levels of PGF2a remained similar in both groups (Table 1). PGs are produced from arachidonic acid release from phospholipids in the cellular membrane by cyclooxygenases (COXs). They are fundamental in generating inflammatory 
responses against pathogens 31,32 . While they have a quick action during the acute phases of the inflammatory response, there is crosstalk with cytokines to synergistically activate NF-kB factor and induce gene expression of pro-inflammatory cytokines and more COXs, mediating positive feedback loops and consequently, chronic inflammation ${ }^{33,34}$.

Since the cellular components that suffer immediate damage are the lipids and proteins of the cell membrane and mitochondrial membrane by lipid peroxidation, the whole-cell physiology is then compromised. One of the cellular mechanisms to revert oxidative stress is the production of several heat shock proteins that repair damage proteins and regulate apoptosis ${ }^{35,36,37}$. A noteworthy result observed in this study was the significant increase $(P<0.01)$ in isoprostane 8-iso-PGF2 $a$ in the jejunum of chickens challenged with $E$. maxima at $7-(P=0.0000252)$ and 9 -days $(P=0.000000717)$ post-challenge compared to the non-challenge control chickens. Excessive generation of reactive oxygen species has been implicated in a variety of pathological events. However, lipid peroxidation is the primary marker of oxidative stress in many pathological conditions, so isoprostanes are reliable biomarkers evaluate it ${ }^{38,39}$. In contrast, F2-isoprostanes (8-Iso-PGF2a) have harmful and potent bioactivities, including vasoconstriction, platelet aggregation, and cardiac hypertrophy $40,41,42,43$. As far as we know, this is the first report of detection of 8-Iso-PGF2a following a challenge of E. maxima in the jejunum (Table 1), despite plasma levels of 8-Iso-PGF2a remaining similar in both groups. It is known that in humans, the plasma half-life of 8-Iso-PGF2 $a$ is one minute at the distribution stage and the removal stage half-life is four minutes ${ }^{44}$. Hence, the half-life in chicken plasma may also be short, which may be why we were not able to detect it. However, pharmacokinetic and metabolic studies evaluating earlier points as well as daily oocyst count are required to confirm and extend these results.

In summary, in the present study, SPF chickens challenged with E. maxima showed an inflammatory response associated with a significant increase (7 days: $P=0.00000268 ; 9$ days: $P=0.00000222$ ) in enteric PGF2a. These changes were related to a significant increase (7 days: $P=0.0000252 ; 9$ days: $P=0.000000717$ ) of enteric 8-Iso-PGF2 $a$ and oocyst excretion, suggesting that the active disease phase was accompanied by inflammation and oxidative stress within the intestinal layer. Since polyunsaturated fatty acids and cholesterol are the principal targets of oxidative stress, lipid peroxidation end products such as 8-Iso-PGF2 $a$ are also part of the pathogenesis of inflammation-related changes caused by $E$. maxima, confirming the role of 8-Iso-PGF2 $a$ as a sensitive biomarker of oxidative stress in chickens. Further studies to control oxidative damage and subsequently intestinal mucosal over-production of lipid oxidation products using phytobiotics, such as essential oils in the diet with specific antioxidants antiinflammatory and coccidicidal properties, are currently being evaluated.

\section{Methods}

\section{Challenge strain}

Eimeria maxima M6 oocysts were provided by Dr. John. R. Barta, University of Guelph, Canada. The methods for detecting and recovering oocysts from challenged chickens, oocyst sporulation, and the 
preparation of infective doses were conducted as described previously ${ }^{45,46}$.

\section{Animal source and experimental design}

Forty one-day-old male SPF chickens (ALPES ${ }^{\circledR}$ Tehuacan, Puebla, Mexico) were randomly allocated to one of two groups with four replicates per group ( $n=5$ chickens/replicate). Chickens were placed in battery cages with a controlled age-appropriate environment at the diagnostic laboratory of the Avian Medicine Department of the Faculty of Veterinary Medicine and Zootechnics (FMVZ) at the National Autonomous University of Mexico (UNAM). Groups consisted of the Control (no challenge) or the Challenge group (E. maxima). Chickens were provided with ad libitum access to water and unmedicated feed. At day 28 of age, all chickens in the challenge group were orally gavaged with 40,000 sporulated $E$. maxima oocysts in a volume of $1 \mathrm{~mL}$ of sterile phosphate-buffered saline solution (PBS). The dose was selected based on a previous trial conducted to determine a challenge dose causing sub-clinical coccidiosis as described previously ${ }^{13}$. Negative control chickens were sham inoculated with $1 \mathrm{~mL}$ of PBS. Seven days after challenge, all chickens were bled, and half of them were euthanized to collect the second half of the jejunum to determine plasma and enteric concentrations of isoprostane 8-iso-PGF2a and PGF2a. At 9 days post-challenge, the rest of the chickens in both groups were bled and jejunum was collected to perform the evaluations. Oocysts per gram (OPG) of feces were evaluated at 7- and 9-days post-challenge.

\section{The standards for 8-iso-PGF2 $a$ and 8-iso-PGF2a-d4}

The standards for 8-iso-PGF2a and 8-iso-PGF2a-d4 (internal standard) were purchased from Cayman Chemicals (Ann Arbor, MI), while the standard for PGF2a was obtained from Sigma-Aldrich (St Louis, MO). Acetonitrile and methanol (HPLC grade) were purchased from JT Baker. Milli-Q water (Millipore system) was used throughout the experiments. Formic acid (FA: 95\%, reactive grade) and isopropanol (LC/MS grade) were purchased from Sigma-Aldrich (St Louis, MO). Ammonium hydroxide $\left(\mathrm{NH}_{4} \mathrm{OH}\right.$, reactive grade, $29.60 \%)$ and potassium hydroxide $(\mathrm{KOH})$ were purchased from JT Baker. For solid-phase microextraction (micro-SPE), 96-well Oasis ${ }^{\circledR}$ MAX $\mu$ Elution cartridges containing a water-wettable reversed-phase strong ammonium exchange mixed-mode polymer, which is selective for acids and stable in organic eluents, were used. A Positive Pressure-96 processor purchased from Waters was also used. Figure 1 shows the chromatograms of standards.

\section{Procedure for the extraction of 8-iso-PGF2 $a$ and PGF2 $a$ in chicken plasma}

Extraction of 8-iso-PGF2 $a$ and PGF2 $a$ were determined as previously described ${ }^{47}$. An aliquot of $500 \mu \mathrm{L}$ chicken plasma was transferred to $2 \mathrm{~mL}$ vials, followed by the addition of $100 \mu \mathrm{L}$ of $4 \mathrm{ng} / \mathrm{mL} 8$-isoPGF2a-d4 as an internal standard and $500 \mu \mathrm{L}$ of hydrolysis solution $(\mathrm{KOH}, 15 \%)$ to release 8-iso-PGF2aesterified. The vials were mixed and incubated in an ultrasonic bath for $30 \mathrm{~min}$ at $40^{\circ} \mathrm{C}$. Subsequently, the vials were cooled to room temperature and $225 \mu \mathrm{L}$ of $6 \mathrm{M}$ formic acid (FA) was added, mixed, and centrifuged at $15000 \mathrm{rpm}$ for $10 \mathrm{~min}$ at $4^{\circ} \mathrm{C}$. Solid-phase microextraction using a 96 -well Oasis ${ }^{\circledR} \mathrm{MAX}$ 
$\mu$ Elution plate conditioned with $500 \mu \mathrm{L}$ of methanol and $500 \mu \mathrm{L}$ of $20 \mathrm{mM}$ FA was used. Finally, the cartridges were loaded with $350 \mu \mathrm{L}$ of plasma and washed with $350 \mu \mathrm{L}$ of $2 \% \mathrm{NH}_{4} \mathrm{OH}$. Samples were then eluted with $50 \mu \mathrm{L}$ of a mixture of 5\% FA in acetonitrile and isopropanol (40:60) and diluted with $150 \mu \mathrm{L}$ of Milli-Q water. Samples were analyzed ( $30 \mu \mathrm{L})$ using ultra-performance liquid chromatography/tandem mass spectrometry (UPLC/MS/MS).

\section{Procedure for the extraction of 8-iso-PGF2 $\alpha$ and PGF2 $\alpha$ in chicken intestine}

For the extraction of 8-iso-PGF2 $\mathrm{a}$ and PGF2a, $0.1 \mathrm{~g}$ of homogenized second half of the jejunum (Meckel's diverticulum to cecal tonsils) were transferred to $2 \mathrm{~mL}$ vials, followed by the addition of $100 \mu \mathrm{L}$ of 4 $\mathrm{ng} / \mathrm{mL}$ 8-iso-PGF2a-d4 as the internal standard and 1.5mL of chloroform: methanol (80:20) mixture. The vials were mixed 30 s by vortex and $15 \mathrm{~min}$ in an ultrasonic bath. Samples were then centrifuged at 15000 rpm for $20 \mathrm{~min}$. The supernatant was evaporated and $500 \mu \mathrm{L}$ of methanol and $500 \mu \mathrm{L}$ of hydrolysis solution (KOH 15\%) were added, mixed, and incubated in an ultrasonic bath for 30 min at $40{ }^{\circ} \mathrm{C}$. Subsequently, the vials were cooled to room temperature and $225 \mu \mathrm{L}$ of $6 \mathrm{M}$ formic acid (FA) and $50 \mu \mathrm{L}$ of $88 \%$ FA were added, mixed, and centrifuged at $15000 \mathrm{rpm}$ for $10 \mathrm{~min}$ at $4{ }^{\circ} \mathrm{C}$. Solid-phase microextraction and analysis of samples were performed in the same way as for the determination of 8-iso-PGF2 $a$ and PGF2 $\alpha$ in chicken plasma using a 96-well Oasis ${ }^{\circledR}$ MAX $\mu$ Elution plate conditioned with $500 \mu \mathrm{L}$ of methanol and $500 \mu \mathrm{L}$ of $20 \mathrm{mM}$ FA. Finally, the cartridges were loaded with $350 \mu \mathrm{L}$ of jejunum sample and washed with $350 \mu \mathrm{L}$ of $2 \% \mathrm{NH}_{4} \mathrm{OH}$. Samples were then eluted with $50 \mu \mathrm{L}$ of a mixture of $5 \% \mathrm{FA}$ in acetonitrile and isopropanol (40:60) and diluted with $150 \mu \mathrm{L}$ of Milli-Q water. The sample $(30 \mu \mathrm{L})$ was injected into a UPLC-MS/MS system for analysis, under the chromatographic and mass spectrometric conditions described previously by Rodriguez Patiño et $a{ }^{47}$.

\section{Ethics}

This study was carried out in accordance with the recommendations for the management of chickens as recommended by the Internal Committee for Care and Use of Experimental Animals (CICUAE, from its abbreviation in Spanish) of the National Autonomous University of Mexico (UNAM), Ethical approval code CICUAE: C20_06, and the study is in compliance with the ARRIVE guidelines where animals are involved.

\section{Quantification of oocysts}

The quantification of OPG from feces was performed at 7- and 9-days post-challenge by using the McMaster technique as previously described ${ }^{45}$.

Data and statistical analysis

PGF2 $a$ and 8-iso-PGF2 $a$ data are presented as means with standard deviation (S.D.). The number of samples per variable group was 20 , implying a normal distribution (Shapiro-Wilk test), and the homoscedasticity was verified (Levene's test). Accordingly, the parametric test of one-tailed Student's t- 
test was performed, and the $P$ value was established with an alpha level of $P<0.01$. OPG data are presented as means with median and variance. The number of samples per variable group was 20; however, the hypotheses of normal distribution (Shapiro-Wilk test) and homoscedasticity (Levene's test) were not confirmed. Consequently, non-parametric tests of the one-tailed Wilcoxon signed-rank test was applied with an alpha level $P<0.05^{48}$.

\section{Declarations}

\section{Acknowledgements}

The research obtained funding from the PAPIIT IT201620 project of DGAPA-UNAM, Universidad Nacional Autónoma de México. The authors thank CONACyT for the doctoral grant number 494367. The research was supported in part by funds provided by USDA-NIFA Sustainable Agriculture Systems, Grant No. 201969012-29905. Title of Project: Empowering U.S. Broiler Production for Transformation and Sustainability USDA-NIFA (Sustainable Agriculture Systems): No. 2019-69012-29905.

\section{Author Contributions}

Conceptualization: VMP-G, RL-A, and GT-I. Investigation and Methodology: GRP, MACR, DH-P, BS-C, and XH-V. Formal analysis and Software: FA-H, CNV, IC-H. Supervision: VMP-G, and GT-I. Validation and Visualization: DH-P and BS-C. Writing-original draft: VP-G and GT-I. Writing-review and editing: $\mathrm{XH}-\mathrm{V}$ and GT-I. All the authors reviewed, edited, and approved the manuscript.

\section{Competing Interests}

The authors declare that the research was conducted in the absence of any commercial or financial relationships that could be construed as a potential conflict of interest.

\section{References}

1. Blake, D. P. et al. Re-calculating the cost of coccidiosis in chickens. Res. 51, 115. doi: 10.1186/s13567-020-00837-2 (2020).

2. Lal, K. et al. Proteomic comparison of four Eimeria tenella life-cycle stages: Unsporulated oocyst, sporulated oocyst, sporozoite and second-generation merozoite. Proteomics 9, 4566-4576. doi: 10.1002/pmic.200900305 (2009).

3. Lillehoj, H., Kim, C., Keeler, C. \& Zhang, S. Immunogenomic approaches to study host immunity to enteric pathogens. Sci. 86, 1491-1500. doi: 10.1093/ps/86.7.1491(2007).

4. Chapman, H. Milestones in avian coccidiosis research: a review. Sci. 93, 501-511. doi: 10.3382/ps.2013-03634 (2014).

5. Allen, P. C. \& Fetterer, R. Recent advances in biology and immunobiology of Eimeria species and in diagnosis and control of infection with these coccidian parasites of poultry. Microbiol. Rev. 15, 58- 
65. doi: 10.1128/cmr.15.1.58-65.2002 (2002).

6. Yun, C., Lillehoj, H. \& Lillehoj, E. Intestinal immune responses to coccidiosis. Comp. Immunol. 24, 303-324. doi: 10.1016/s0145-305x(99)00080-4 (2000).

7. Kim, W. H., Chaudhari, A. A. \& Lillehoj, H. S. Involvement of T cell immunity in avian coccidiosis. Immunol. 10, 2732. doi: 10.3389/fimmu.2019.02732 (2019).

8. Hong, Y. H., Lillehoj, H. S., Lee, S. H., Dalloul, R. A. \& Lillehoj, E. P. Analysis of chicken cytokine and chemokine gene expression following Eimeria acervulina and Eimeria tenella Vet. Immunol. Immunopathol. 114, 209-223. doi: 10.1016/j.vetimm.2006.07.007 (2006).

9. Hong, Y.H., Lillehoj, H.S., Lillehoj, H.P. \& Lee, S.H. Changes in immune-related gene expression and intestinal lymphocyte subpopulations following Eimeria maxima infection of chickens. Immunol. Immunopathol. 114, 259-272. doi: 10.1016/j.vetimm.2006.08.006 (2006).

10. Lillehoj, H., Min, W. \& Dalloul, R. Recent progress on the cytokine regulation of intestinal immune responses to Eimeria. Sci. 83, 611-623. doi: 10.1093/ps/83.4.611 (2004).

11. Lillehoj, H. S. \& Trout, J. M. Avian gut-associated lymphoid tissues and intestinal immune responses to Eimeria Clin. Microbiol. Rev. 9, 349-360. doi: 10.1128/CMR.9.3.349-360.1996 (1996).

12. Park, S. S. et al. Immunopathology and cytokine responses in broiler chickens coinfected with Eimeria maxima and Clostridium perfringens with the use of an animal model of necrotic enteritis. Avian Dis. 52, 14-22. doi: 10.1637/7997-041707-Reg (2008).

13. Tomley, F. M., Clarke, L. E., Kawazoe, U., Dijkema, R. \& Kok, J. J. Sequence of the gene encoding an immunodominant microneme protein of Eimeria tenella. Biochem. Parasitol. 49, 277-288. doi: 10.1016/0166-6851(91)90071-d (1991).

14. Tomley, F. M., Billington, K. J., Bumstead, J. M., Clark, J. D. \& Monaghan, P. EtMIC4: a microneme protein from Eimeria tenella that contains tandem arrays of epidermal growth factor-like repeats and thrombospondin type-I repeats. J. Parasitol. 31, 1303-1310. doi: 10.1016/s0020-7519(01)00255-7 (2001).

15. Zhou, Z. et al. Upregulation of chicken TLR4, TLR15 and MyD88 in heterophils and monocyte-derived macrophages stimulated with Eimeria tenella in vitro. Parasitol. 133, 427-433. doi: 10.1016/j.exppara.2013.01.002 (2013).

16. Crane, J. K. \& Mongiardo, K. M. Pro-inflammatory effects of uric acid in the gastrointestinal tract. Invest. 43, 255-266. doi: 10.3109/08820139.2013.864667 (2014).

17. Sellin, M.E., Müller, A. A. \& Hardt, W-D. Consequences of epithelial inflammasome activation by bacterial pathogens. Mol. Biol. 430, 193-206. doi: 10.1016/j.jmb.2017.03.031 (2018).

18. Hornung, V. et al. AIM2 recognizes cytosolic dsDNA and forms a caspase-1-activating inflammasome with ASC. Nature 458, 514-518. doi: 10.1038/nature07725 (2009).

19. Jiang, L. et al. Identification and characterization of Eimeria tenella apical membrane antigen-1 (AMA1). PLoS One 7, e41115. doi: 10.1371/journal.pone.0041115 (2012). 
20. Soldati, D., Foth, B. J. \& Cowman, A. F. Molecular and functional aspects of parasite invasion. Trends Parasitol. 20, 567-574. doi: 10.1016/j.pt.2004.09.009 (2004).

21. Dowse, T. \& Soldati, D. Host cell invasion by the apicomplexans: the significance of microneme protein proteolysis. Opin. Microbiol. 7, 388-396. doi: 10.1016/j.mib.2004.06.013 (2004).

22. Russell, D. Host cell invasion by Apicomplexa: an expression of the parasite's contractile system? Parasitology 87, 199-209. doi: 10.1017/s0031182000052562 (1983).

23. Duszenko, M. et al. Autophagy in protists. Autophagy 7, 127-158. doi: 10.4161/auto.7.2.13310 (2011).

24. Nouwen, L. V. \& Everts B. Pathogens MenTORing macrophages and dendritic cells: manipulation of mTOR and cellular metabolism to promote immune escape. Cel/s 9, 161. doi: 10.3390/cells9010161 (2020).

25. Escalante, A. A. \& Ayala, F. J. Evolutionary origin of Plasmodium and other Apicomplexa based on rRNA genes. Natl. Acad. Sci. U.S.A. 92, 5793-5797. doi: 10.1073/pnas.92.13.5793 (1995).

26. Lillehoj, H. S. \& Li, G. Nitric oxide production by macrophages stimulated with coccidia sporozoites, lipopolysaccharide, or interferon-a, and its dynamic changes in SC and TK strains of chickens infected with Eimeria tenella. Avian Dis. 48, 244-253. doi: 10.1637/7054 (2004).

27. Lauridsen, C. From oxidative stress to inflammation: redox balance and immune system. Poult. Sci. 98, 4240-4246. doi: 10.3382/ps/pey407 (2019).

28. Gebicki, J. M. Oxidative stress, free radicals, and protein peroxides. Arch. Biochem. Biophys. 595, 3339. doi: 10.1016/j.abb.2015.10.021 (2016).

29. Cadenas, E., Packer, L. \& Traber, M. G. Antioxidants, oxidants, and redox impacts on cell function-A tribute to Helmut Sies-. Biochem. Biophys. 595, 94-99. doi: 10.1016/j.abb.2015.11.01 (2016).

30. Yodoi, J., Tian, H., Masutani, H. \& Nakamura, H. Thiol redox barrier; local and systemic surveillance against stress and inflammatory diseases. Biochem. Biophys. 595, 88-93. doi: 10.1016/j.abb.2015.11.029 (2016).

31. Ricciotti, E. \& FitzGerald, G. A. Prostaglandins and inflammation. Thromb. Vas. Biol. 31,986-1000. doi: 10.1161/ATVBAHA.110.207449 (2011).

32. Yao, C. \& Narumiya, S. Prostaglandin-cytokine crosstalk in chronic inflammation. J. Pharmacol. 176, 337-354. doi: 10.1111/bph.14530 (2019).

33. Zhao, G. et al. Pivotal role of reactive oxygen species in differential regulation of lipopolysaccharideinduced prostaglandins production in macrophages. Pharmacol. 83, 167-178. doi: 10.1124/mol.112.080762 (2013).

34. Wang, D. \& DuBois RN. Role of prostanoids in gastrointestinal cancer. Clin. Invest. 128, 2732-2742 (2018).

35. Zeng, T. et al Effects of heat stress on antioxidant defense system, inflammatory injury, and heat shock proteins of Muscovy and Pekin ducks: evidence for differential thermal sensitivities. Cell Stress Chaperones 19:895-901. doi: 10.1007/s12192-014-0514-7 (2014). 
36. Xu, J., Tang, S., Song, E., Yin, B., Bao, E. Inhibition of heat shock protein 70 intensifies heat-stressed damage and apoptosis of chicken primary myocardial cells in vitro. Med. Rep. 15, 2881-2889. doi: 10.3892/mmr.2017.6337 (2017).

37. Çenesiz, The role of oxidant and antioxidant parameters in the infectious diseases: a systematic literature review. Kafkas Üniv. Vet. Fak. Derg. 26,849-858. doi: 10.9775/kvfd.2020.24618 (2020).

38. Milne, G. L., Dai, Q. \& Roberts, II L. J. The isoprostanes-25 years later. Biophys. Acta 1851, 433-445. doi: 10.1016/j.bbalip.2014.10.007 (2015).

39. Czerska, M., Zieliński, M. \& Gromadzińska, J. Isoprostanes-A novel major group of oxidative stress markers. J. Occup. Med. Environ. Health 29, 179-190. doi: 10.13075/ijomeh.1896.00596 (2016).

40. Wen, S-H. et al. Role of 15-F2t-isoprostane in intestinal injury induced by intestinal ischemia/reperfusion in rats. Free Radic. Res. 48, 907-918. doi: 10.3109/10715762.2014.926010 (2014).

41. Crankshaw, D. \& Rangachari, P. Isoprostanes: more than just mere markers. Cell. Biochem. 253, 125130. doi: 10.1023/a:1026052123843 (2003).

42. Kaviarasan, S., Muniandy, S., Qvist, R. \& Ismail, I. S. F2-isoprostanes as novel biomarkers for type 2 diabetes: a review. Clin. Biochem. Nutr. 45, 1-8. doi: 10.3164/jcbn.08-266 (2009).

43. Janicka, M., Kot-Wasik, A., Kot, J. \& Namie'snik, J. Isoprostanes-biomarkers of lipid peroxidation: their utility in evaluating oxidative stress and analysis. J. Mol. Sci. 11, 4631-4659. doi: 10.3390/ijms11114631 (2010).

44. Basu, S. Metabolism of 8-iso-prostaglandin F2a. FEBS Lett 428, 32-36. doi: 10.1016/s00145793(98)00481-5 (1998).

45. Long, P. L., Millard, B. J., Joyner, L. P. \& Norton, C. C. A guide to laboratory techniques used in the study and diagnosis of avian coccidiosis. Folia Vet. Lat. 6, 201-217 (1976).

46. Haug, A., Williams, R. \& Larsen, S. Counting coccidial oocysts in chicken faeces: a comparative study of a standard McMaster technique and a new rapid method. Parasitol. 136, 233-242. doi: 10.1016/j.vetpar.2005.11.024 (2006).

47. Rodriguez Patiño, G. et al. Development of a method for the determination of 8-iso-PGF2a in sheep and goat plasma using solid-phase microextraction and ultra-performance liquid chromatography/tandem mass spectrometry. Rapid Commun. Mass Spectrom. 32, 1675-1682. doi: 10.1002/rcm.8224 (2018).

48. SAS Institute Inc. SAS/STAT® 9.2 User's Guide. 3530-3541. (SAS Institute Inc, 2009).

\section{Tables}

Table 1. Evaluation of isoprostane 8-iso-PGF2 $\alpha$ and prostaglandin GF2 $\alpha$ from enteric (jejunum) and plasma of SPF chickens challenged with Eimeria maxima at 7 and 9 days postchallenge. 


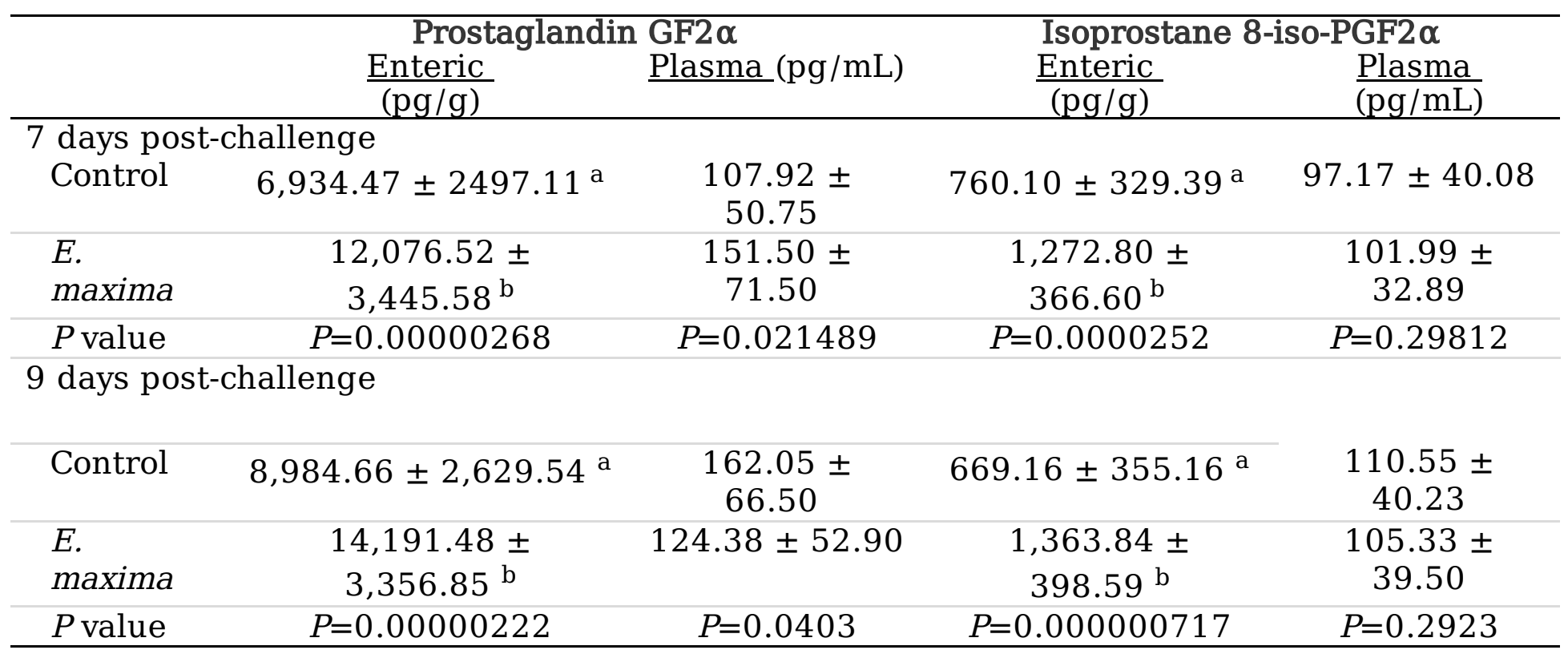

Data expressed as mean \pm standard deviation. $n=20$.

${ }^{\mathrm{ab}}$ Different superscripts within columns and days indicate a significant difference at $P<0.01$.

Table 2. Eimeria maxima oocyst per gram in the feces of SPF chickens at 7 and 9 days postchallenge.

\begin{tabular}{|c|c|c|c|}
\hline & 7 days post-challenge & 9 days post-challenge & $\mathrm{y}, \mathrm{z} P$ value \\
\hline Control & $0 \pm 0^{\text {a.y }}$ & $0 \pm 0^{\text {a.y }}$ & $P=0.50$ \\
\hline E. maxima & $24,240(20,212.5 ; 0.37868) b, y$ & $2,750(1,700,0.823027) \mathrm{b}, \mathrm{z}$ & $P=0.00002$ \\
\hline${ }^{\mathrm{a}, \mathrm{b}} P$ value & $P=0.00000095$ & $P=0.00000095$ & \\
\hline
\end{tabular}

Each value represents the mean (median; variance). $\mathrm{n}=20$.

${ }^{\mathrm{a}-\mathrm{b}}$ Values within groups columns, or ${ }^{\mathrm{y}-\mathrm{z}}$ values within time of evaluation within columns with different superscripts differ significantly at $P<0.05$.

\section{Figures}




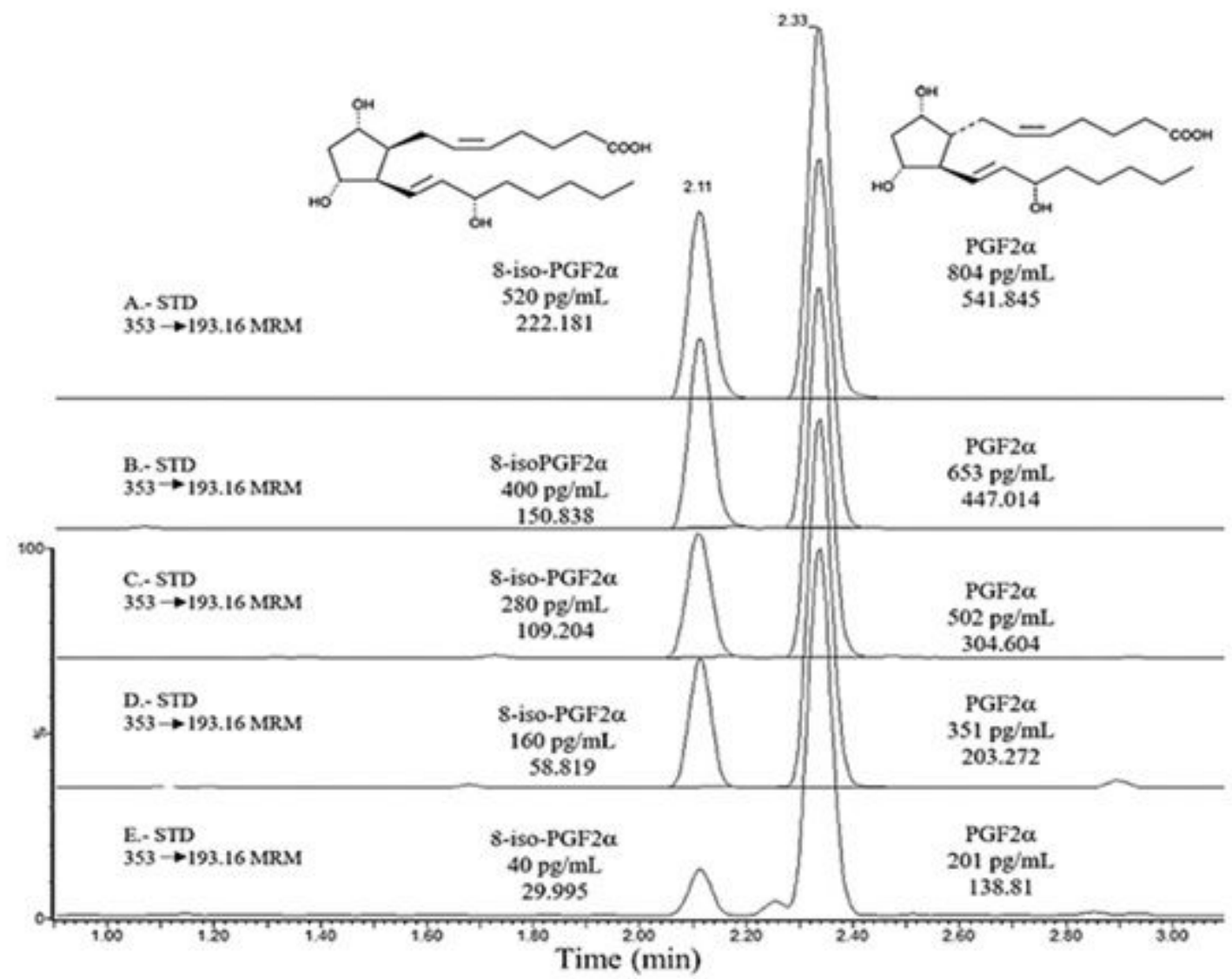

\section{Figure 1}

Chromatograms of standards. Mix of 8-iso-PGF2 $a$ and PGF2a: A.- 520 pg of 8-iso-PGF $2 a / m L$ and $541.845 \mathrm{pg}$ of PGF2a/mL, B.- $400 \mathrm{pg}$ of 8-iso-PGF2a/mL and $653 \mathrm{pg}$ of PGF2 $\mathrm{a} / \mathrm{mL}$, C.- $280 \mathrm{pg}$ of 8-isoPGF2a/mL and $502 \mathrm{pg}$ of PGF2a/mL, D.- $160 \mathrm{pg}$ of 8-iso-PGF2 $\mathrm{a} / \mathrm{mL}$ and $351 \mathrm{pg}$ of PGF $2 \mathrm{a} / \mathrm{mL}$, and E.- 40 $\mathrm{pg}$ of 8-iso-PGF2a/mL and $201 \mathrm{pg}$ of PGF2a/mL. 

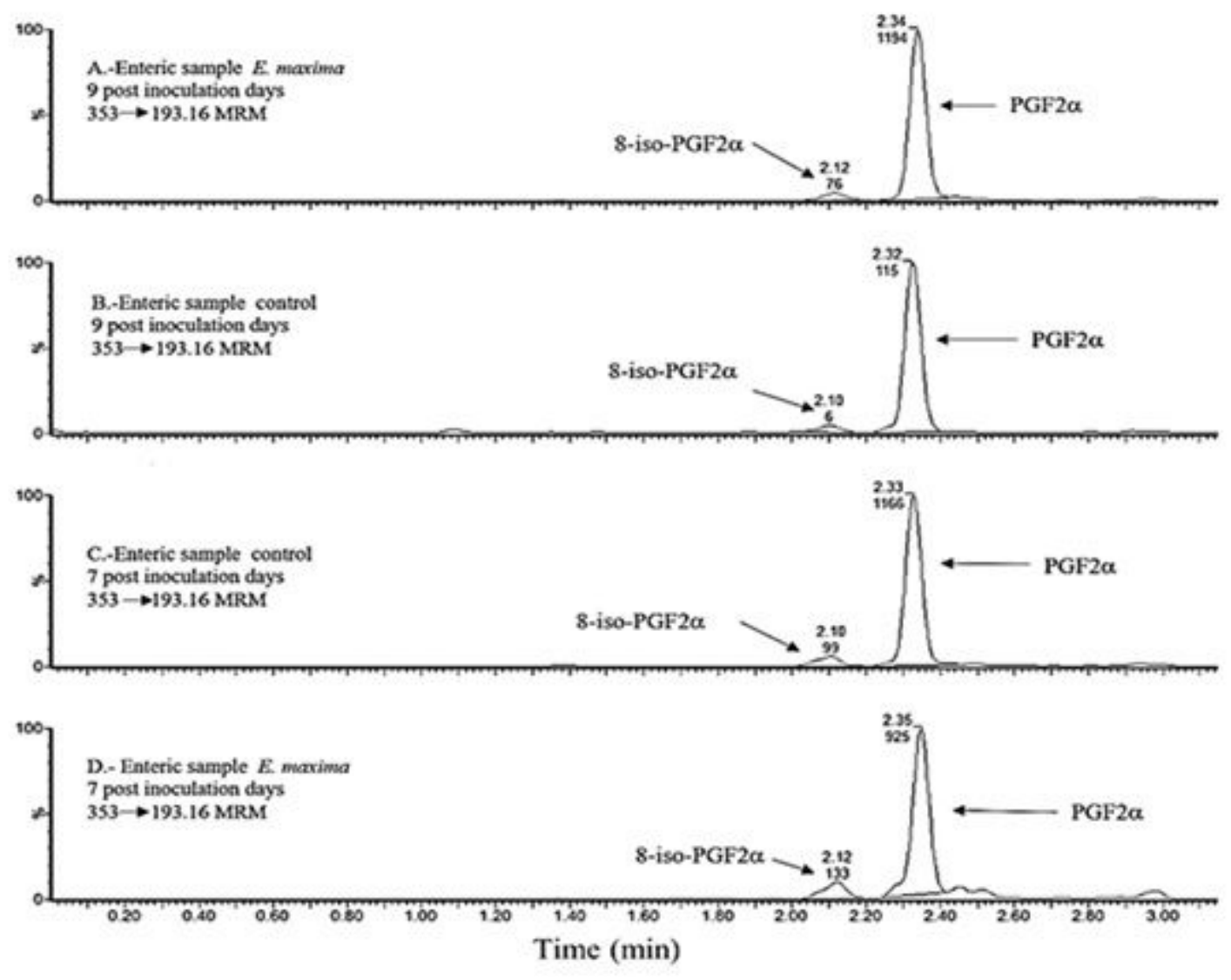

Figure 2

Chromatograms of 8-iso-PGF2a and PGF2a obtained from enteric (jejunum) samples: A.- sample of E. maxima 9 days post-inoculation chickens, B.- sample of control of 9 days post-inoculation chickens, C.sample of control of 7 days post-inoculation chickens, and D.- sample of E. maxima 7 days postinoculation chickens. 

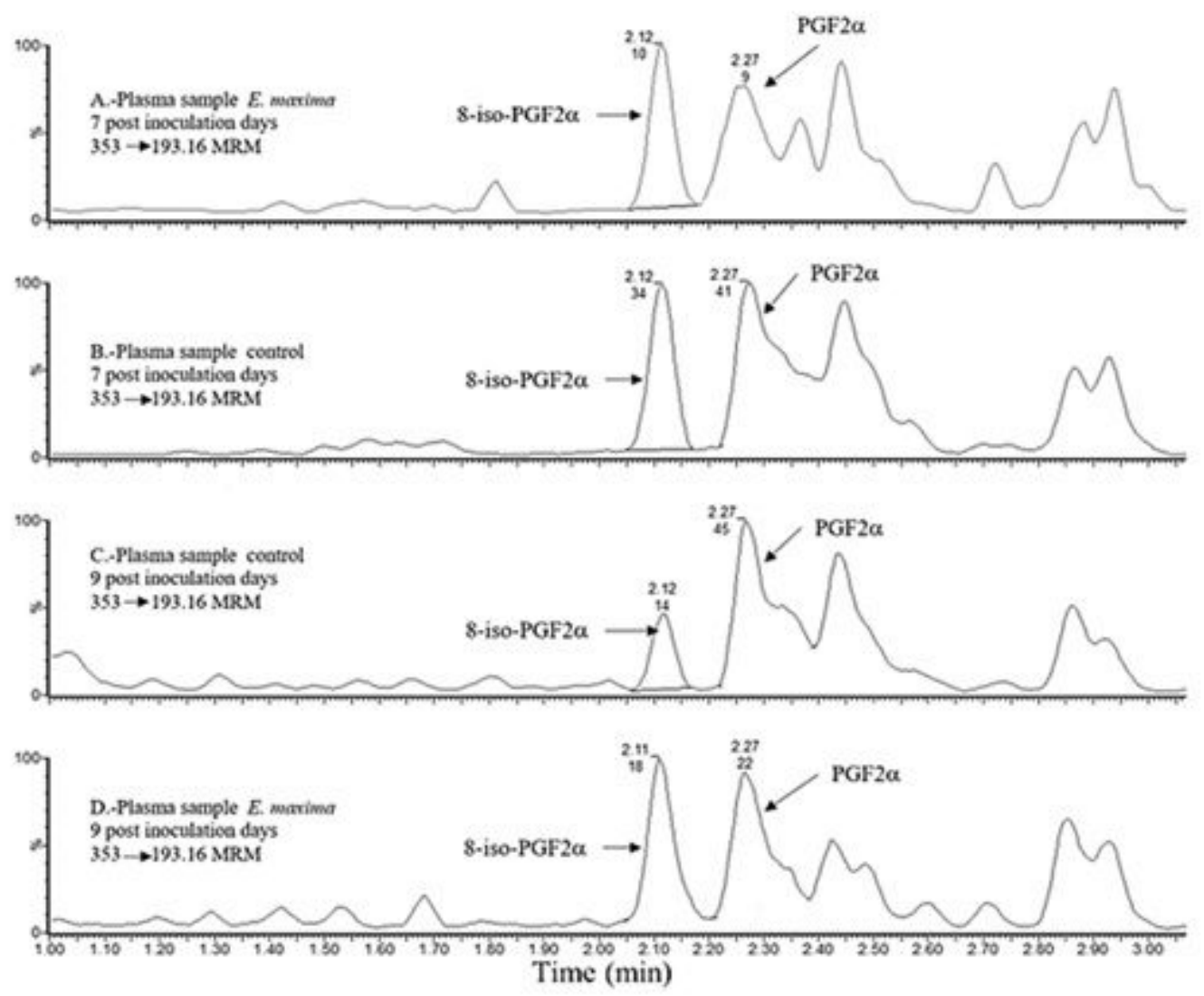

\section{Figure 3}

Chromatograms of standards of 8-iso-PGF2 $a$ and PGF2 $a$ in plasma samples: A.- sample of E. maxima 7 days post-inoculation chickens, B.- sample of control of 7 days post-inoculation chickens, C.- sample of control of 9 days post-inoculation chickens, and D.- sample of E. maxima 9 days post-inoculation chickens. 